\title{
Reversible SA nodal dysfunction
}

Sinus node dysfunction is a well-known entity since almost a century. However, predominantly considered as a genetic, degenerative or an infiltrative process, it's only recently that ischemia as an etiology has come to the forefront. This is a case illustrating reversible SA nodal dysfunction due to ischemia. As soon as occluded RCA was opened the patient recovered sinus rhythm. However, further research is needed to understand the relationship of schema with SA nodal dysfunction and which ones would revert to sinus rhythm on revascularisation.

\section{Introduction}

Sinus node dysfunction refers to the pathologic affection of the sinus node. When symptomatic it is referred to as the sick sinus syndrome. First described by Dr. M. Irene Ferrer has myriad manifestations ranging from persistent bradycardia, sinus arrest, tachy-brady syndrome and junctional rhythm [1]. Intrinsic causes of sick sinus syndrome include degenerative fibrosis of the sinoatrial node, ion channel dysfunction, and remodeling of the sinoatrial node. Historically, the most common intrinsic cause is thought to be age-related, idiopathic degenerative fibrosis of the sinoatrial node. Recent research and understanding of familial and congenital sick sinus syndromes, however, have shown that an inherited dysfunction of ion channels within the sinoatrial node also plays a significant part in age-related sick sinus syndrome [2]. In recent years, it is being realised that there are reversible causes of sick sinus syndrome.

The blood supply of SA node is complex. The artery that supplies the SA node (ramus nodi sinuatrialis) was a branch of either the right or the left coronary artery. There was no relationship between topographic coronary dominance and the origin of the SA node artery. Most frequently (in 63\% of cases), the SA node artery was the first anterior atrial branch of the right coronary artery (Figure 1),
Kumar D*, Pawar A, Sabnis G, Shah H, Lanewar C, Kerkar P, More D

Department of Cardiology, King Edward Memorial Hospital Mumbai, Maharashtra, India

*Author for correspondence:

Tel.: +91-9323361116

dhiraj1645@gmail.com

Submitted: March 21, 2018

Accepted: April 23, 2018

Published online: April 30, 2018
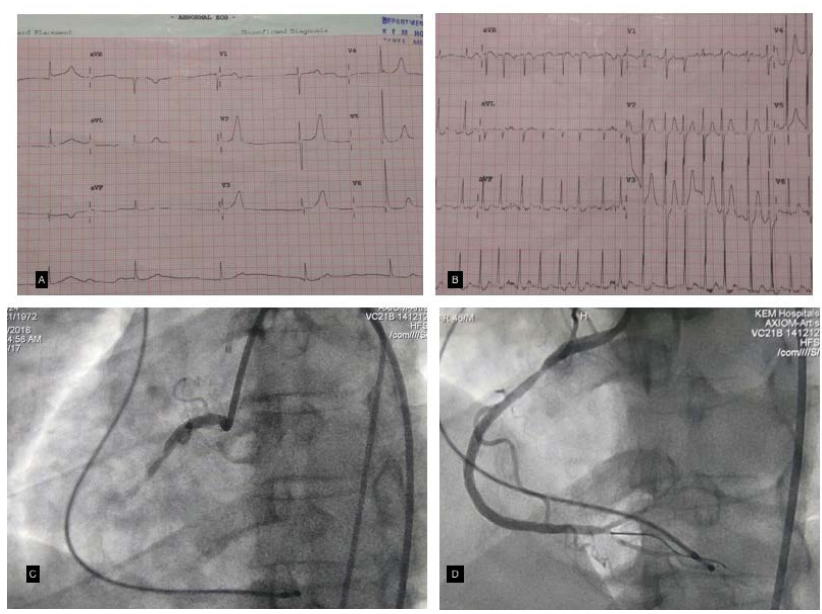

Figure 1: A) Junctional rhythm with escape rate of 38/min B) Transient Atrial Fibrillation C) Mid RCA total occlusion D) Post balloon dilation. 
originating at a distance of $1.2 \mathrm{~cm}$ (range $0.2-2.2 \mathrm{~cm}$ ) from the beginning of this vessel. In certain cases (37\%), the artery to the SA node is a branch of the left coronary artery. It most frequently arises from the proximal portion of the left circumflex branch ( $95 \%$ of cases) and sometimes directly from the left coronary artery (3\% of cases) [3].

Although it is known that ischemia can lead to sinus node dysfunction there is not enough data to suggest whether it is reversible or not [4].

\section{Case Report}

We present a case in which a 47-year-old male who had been hypertensive and diabetic and presented with syncope while working at home and came to the ER where he was found to have junctional rhythm (Figure 1A). The patient was shifted to catheterisation laboratory and had a transient episode of atrial fibrillation with a controlled ventricular rate (Figure 1B). After temporary pacing patient was found to have right ventricular and right atrial dilation and normal left ventricular function. High sensitivity quantitative troponin I of 699 (upper limit of 19). An angiogram was carried out next day which was suggestive of midRCA total occlusion with a narrowed SA nodal branch (Figure 1C). The lesion was first pre-dilated with a semi-compliant balloon (Figure 1D) and then stenting was done with drug-eluting stent (Figure 2A). Post PCI the patient was in sinus rhythm (Figure 2B).

\section{Discussion}

Sick sinus syndrome is often a disease of older agegroup. However, increasingly younger aged people are being diagnosed with this disorder, especially genetic aetiology. However, ischemia is emerging as an important cause and whenever a young patient presents with a rhythm disorder then ischemia as a cause should

\section{References}

1. Ewy GA. Sick Sinus Syndrome Synopsis. J Am Coll Cardiol. 64: 539-40 (2014).

2. Jensen PN, Gronroos NN, Chen LY, et al. Incidence of and risk factors for sick sinus syndrome in the general population. $\mathrm{J} \mathrm{Am}$ Coll Cardiol. 64: 531-8 (2014).

3. B Pejovic, I Krajnc, F Anderhuber, et al. Anatomical Aspects of the Arterial Blood Supply to the Sinoatrial and
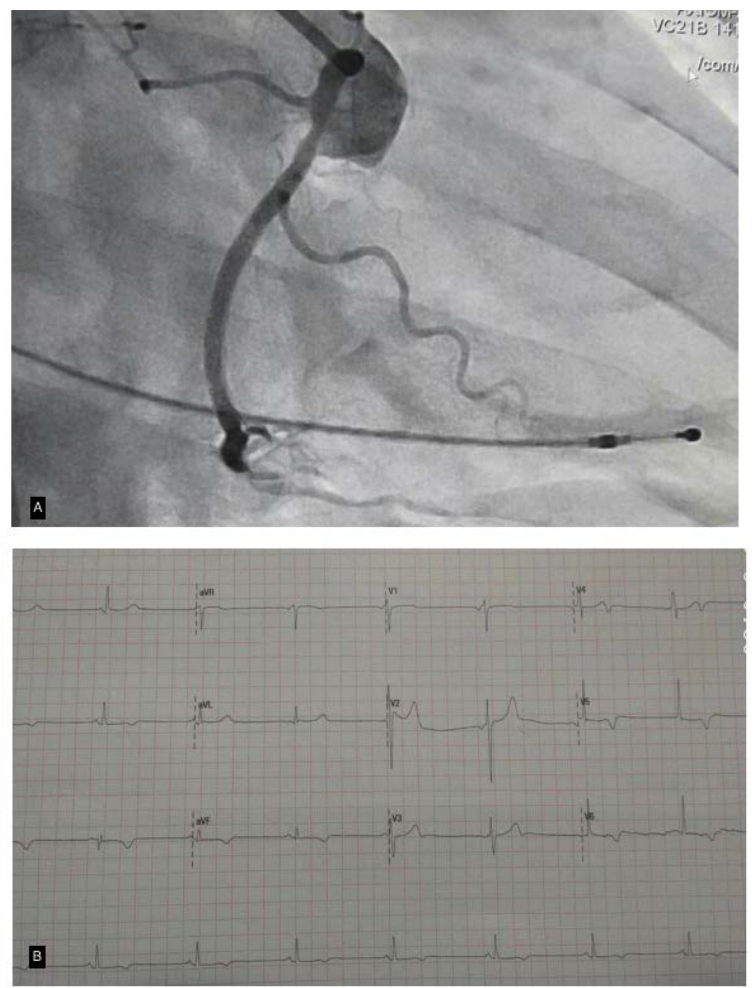

Figure 2: A) Post $\mathrm{PCl}$ prominent $\mathrm{SA}$ nodal artery B) Post $\mathrm{PCl}$ sinus rhythm ecg.

be ruled out as it can be reversible and save pacemaker implantations. However, whether the patients do recover totally or ultimately still need a pacemaker still needs to be resolved [5].

\section{Conclusion}

Ischemia is not an infrequent cause of sinus node dysfunction. It may be reversible if revascularisation is done early enough. Therefore, even in patients who may present with subtle symptoms of ischemia angiogram must be performed.
Atrioventricular Nodes of the Human Heart. I Int Med Res. 36(4):691-8 (2008).

4. Parameswaran R, Ohe T, Goldberg H. Sinus node dysfunction in acute myocardial infarction. Br Heart J. 38(1): 93-96 (1976).

5. Epstein AE, DiMarco JP, Ellenbogen KA, et al. ACC/AHA/ HRS 2008 guidelines for device-based therapy of cardiac rhythm abnormalities: a report of the American College of Cardiology/ American Heart Association Task Force on Practice. J Am Coll Cardiol. 51: e1-62 (2008). 